the perturbation from the first solvent layer. These calculations then suggest that the vibrational dephasing is mainly determined by the average force field that the excited molecules experience from the fluctuating number of nearest neighbors. Also in agreement with this picture is the increse in the correlation time (Table I) with increasing molecular interaction. ${ }^{17}$

The investigations were supported in part by the Netherlands Foundation for Chemical Research (SON) with financial aid from the Netherlands Organization for the Advancement of Pure Research (ZWO).

\footnotetext{
${ }^{1}$ D. von der Linde, A. Laubereau, and W. Kaiser, Phys. Rev. Lett. 26, 954 (1971).

${ }^{2}$ S. F. Fisher and A. Laubereau, Chem. Phys. Lett. $\underline{35}, 6$ (1975).

${ }^{3}$ A. Laubereau, Chem. Phys. Lett. 27, 600 (1974).

${ }^{4}$ A. Laubereau, G. Wochner, and W. Kaiser, Phys.
}

Rev. A 13, 2212 (1976).

${ }^{5}$ D. J. Diestler, Chem. Phys. Lett. $\underline{39}, 39$ (1976).

${ }^{6}$ D. W. Oxtoby and S. A. Rice, Chem. Phys. Lett. $\underline{42}$, 1 (1976).

${ }^{7}$ W. G. Rothschild, J. Chem. Phys. 65, 2958 (1976).

${ }^{8}$ A. Rahman, Phys. Rev. 136, 405 (1964).

${ }^{9} \mathrm{~J}$. A. Giordmaine and W. Kaiser, Phys. Rev. 144, 676 (1966).

${ }^{10}$ W. F. Calaway and G. E. Ewing, Chem. Phys, Lett. $\underline{30,} 485$ (1975).

${ }^{11}$ Y. le Duff, J. Chem. Phys. $\underline{59}, 1984$ (1973).

${ }^{12} \mathrm{M}$. Scotto, J. Chem. Phys. $\underline{49}, 5362$ (1968).

${ }^{13} \mathrm{R}$. Kubo, in Fluctuations, Relaxation and Resonance in Magnetic Systems, edited by D. Ter Haar (Plenum, New York, 1962), p. 27.

${ }^{14}$ S. Bratos and E. Marechal, Phys. Rev. A $\underline{4}, 1078$ (1971).

${ }^{15}$ P. S. Y. Cheung and J. G. Powless, Mol. Phys. $\underline{30}$, 921 (1975).

${ }^{16}$ B. Quentrec and C. Brot, Phys. Rev. A $\underline{12}, 272$

(1975).

${ }^{17}$ K. Toukubo and K. Nakanishi, J. Chem. Phys. $\underline{65}$, 1937 (1976).

\title{
Colliding Impulsive Gravitational Waves
}

\author{
Y. Nutku \\ Joseph Henry Laboratories, Princeton University, Princeton, New Jersey 08540, and \\ Department of Physics, Middle East Technical University, Ankara, Turkey \\ and \\ M. Halil \\ Department of Physics, Middle East Technical University, Ankara, Turkey \\ (Received 18 July 1977)
}

\begin{abstract}
We formulate the problem of colliding plane gravitational waves with two polarizations as the harmonic mappings of Riemannian manifolds and construct an exact solution of the vacuum Einstein field equations describing the interaction of colliding impulsive gravitational waves which in the limit of collinear polarization reduces to the solution of Khan and Penrose.
\end{abstract}

In this Letter we adopt an approach to the Einstein field equations of gravitation which is based on the theory of Eells and Sampson of harmonic mappings of Riemannian manifolds. ${ }^{1}$ We had earlier pointed out the connection between these problem $^{2,3}$ and it seems worthwile to remark that the theory of harmonic mappings of Riemannian manifolds is also applicable to a wide variety of problems in other branches of physics. In particular, we can readily recognize the Nambu string, solitons, nonlinear $\sigma$ model, and the Heisenberg ferromanget in the expression of Eells and Sampson for their invariant functionals of the mapping. The formulation of a problem in terms of harmonic mappings provides us with a powerful formalism for the discussion of a num- ber of questions ranging from the construction of exact solutions to considerations of topology related to the index of the mapping, and its advantage lies in the direct geometrical insight it brings into the problem. We shall now present a new exact solution of the vacuum Einstein equations which is of physical interest and which was obtained by the use of these techniques.

Penrose $^{4}$ has introduced the notion of impulsive gravitational waves where space-time is flat everywhere except along a hypersurface with the Riemann tensor suffering a $\delta$-function discontinuity at this surface. Here we shall be concerned with the case where the discontinuity surface is a null plane and the impulsive wave is then a familiar $p-p$ wave. ${ }^{5}$ For purely impulsive gravitational 
waves the surface of discontinuity is shear-free; however, when two impulsive waves collide they produce shear and the mutual focusing and backscattering of these waves results in a space-time singularity. This picture emerges from the exact solution of Khan and Penrose ${ }^{6}$ which describes colliding impulsive plane gravitational waves with collinear polarization. It illustrates the necessity of working with exact solutions of the Einstein field equations since, as Szekeres ${ }^{7,8}$ has shown, arbitrarily weak incoming gravitational waves inevitably produce a space-time singularity. In this Letter we shall present an exact solution of the vacuum Einstein field equations which enables us to study the geometry of space-time resulting from the collision of two linearly polarized impulsive waves with arbitrary relative polarization. We find that in the interaction region the solution is no longer linearly polarized and the focusing properties ${ }^{9}$ of the waves result in the development of an angular-momentum as well as a mass as- pect, but once again the collision produces a spacelike curvature singularity on the space-time manifold.

Impulsive gravitational plane waves are described by the metric for $p-p$ waves

$$
\begin{aligned}
& d s^{2}=2 d u^{\prime} d v^{\prime}-d x^{\prime 2}-d y^{\prime 2}-2 H\left(x^{\prime}, y^{\prime}\right) \delta\left(u^{\prime}\right) d u^{\prime}, \\
& H\left(x^{\prime}, y^{\prime}\right)=\frac{1}{2}\left(x^{\prime 2}-y^{\prime 2}\right) \cos \alpha+x^{\prime} y^{\prime} \sin \alpha,
\end{aligned}
$$

where $\alpha$ is a constant which measures the angle of polarization. In Eq. (2) $H$ has been scaled by a constant factor to set the amplitude of the wave equal to unity. This can be accomplished without loss of generality since such a constant can always be restored by a coordinate transformation. The coordinate patch used in writing the metric (1) has the advantage of manifestly displaying the Minkowski form of the metric on both sides of the discontinuity surface, but for reasons which are discussed in detail by Szekeres ${ }^{8}$ it is inappropriate for the colliding waves problem. For this purpose we transform to the Rosen form

$$
d s^{2}=2 e^{-M} d u d v-e^{-U}\left(e^{V} \cosh W d x^{2}-2 \sinh W d x d y+e^{-V} \cosh W d y^{2}\right)
$$

which has two mutually nonorthogonal Killing vectors $\vec{\xi}_{x}$ and $\vec{\xi}_{y}$ and where the metric coefficients are $C^{0}-$ differentiable functions of the null coordinates $u, v$. The transformation which accomplishes this task is given by

$$
\begin{aligned}
& x^{\prime}=[1+u \theta(u) \cos \alpha] x+[u \theta(u) \sin \alpha] y, \\
& y^{\prime}=[1-u \theta(u) \cos \alpha] y+[u \theta(u) \sin \alpha] x, \\
& u^{\prime}=u, \\
& v^{\prime}=v+\left[\frac{1}{2}(\cos \alpha)\left(x^{2}-y^{2}\right)+(\sin \alpha) x y\right] \theta(u)+\frac{1}{2}\left(x^{2}+y^{2}\right) u \theta(u),
\end{aligned}
$$

where $\theta$ is the Heaviside unit step function. Now it can be readily verified that the metric (1) is in the form of Eq. (3) with

$$
\begin{aligned}
& e^{-U}=1-p^{2}, \\
& e^{2 V}=\frac{\left(1+2 p \cos \alpha+p^{2}\right)}{\left(1-2 p \cos \alpha+p^{2}\right)}, \\
& \sinh W=-2 p \sin \alpha /\left(1-p^{2}\right), \\
& M=0,
\end{aligned}
$$

with the conventional definition

$$
p=u \theta(u) \text {. }
$$

In the region II the metric for the incoming gravitational wave will be given by Eqs. (3) and (5). There will be a similar expression for the metric in region III if we replace all the $p$ 's in Eq. (5) with

$$
q=v \theta(v),
$$

and the polarization angle $\alpha$ by another angle which will be called $\beta$. In this situation the waves in regions II and III are linearly polarized but their polarizations differ by the angle $\alpha-\beta$. The collision problem is then formulated as follows: We consider two gravitational waves traveling in $+z$ and $-z$ directions. Before the collision, the region between them (I) is a Minkowski space and for impulsive waves region II $\{u>0, v<0\}$ and region III $\{u<0, v>0\}$ are flat as well. The junction conditions between regions I and II, and between I and III, are the usual impulsive wave conditions. In region IV the two waves collide head-on and their interaction is determined by the Einstein field equations for the metric (3) 
with the requirement that the solution reduces to the known solutions in regions II and III [cf. Eq. (5)], as well as satisfying the necessary junction conditions across the various regions. The Ricci tensor will be zero throughout. The Einstein field equations for this problem are well known, but we shall now present a new formulation of these equations using the theory of harmonic mappings of Riemannian manifolds which brings an element of simplicity into the problem.

We consider two Riemannian manifolds $(M, g)$ and $\left(M^{\prime}, g^{\prime}\right)$ with a mapping $f: M \rightarrow M^{\prime}$. An invariant functional of this mapping is the trace with respect to the metric $g$ of the induced metric $f^{*} g^{\prime}$ on $M$. The integral of this quantity over the volume of $M$ is the Eells-Sampson energy functional $E(f)$. In local coordinates it is given by

$$
E(f)=\int g_{A B}{ }^{\prime} \frac{\partial f^{A}}{\partial x^{i}} \frac{\partial f^{B}}{\partial x^{k}} g^{i k} \sqrt{g} d^{n} x,
$$

and those maps for which the first variation vanishes,

$$
\delta E(f)=0,
$$

are called harmonic. The Einstein field equations for the metric in Eq. (3) are obtained as harmonic maps if we consider the following two Riemannian manifolds: Let $M$ be a flat two-dimensional manifold with the metric

$$
d s^{2}=2 d u d v
$$

and consider $M^{\prime}$ to be endowed with the metric

$$
d s^{\prime 2}=e^{-U}\left(2 d M d U+d U^{2}-d W^{2}-\cosh ^{2} W d V^{2}\right)
$$

It should be noted that the metrics (10) and (11) are given known metrics, and even though we wrote them using particular coordinate patches, it is evident that their significance is not tied to any coordinate system. Now, if we form the energy functional (8) using the metrics (10) and (11), we obtain the Hilbert action principle specialized to the metric (3) and the condition for the mapping to be harmonic is equivalent to satisfaction of the Einstein field equations.

The formulation of the Einstein field equations as the problem of the harmonic mappings of
Riemannian manifolds is essentially a "mini-superspace"10 approach; indeed, it grew out of many stimulating conversations with Professor C. W. Misner. It is helpful in constructing exact solutions because the requirement that the mapping $f: M-M^{\prime}$ be harmonic is an invariant statement which is not affected by any choice of coordinates on $M$ and $M^{\prime}$. Thus by performing coordinate transformations on $\boldsymbol{M}$ and $M^{\prime}$ we can obtain all possible forms of the Einstein field equations which respect the choices made earlier about the Killing directions in the space-time manifold. They will furthermore be consistent with the freedom inherited from general covariance and the arbitrariness available in choosing any Ansatz about the form of the nonvanishing metric coefficients in the original space-time metric. It is therefore natural to look for coordinate transformations on $M$ and $M^{\prime}$ such that the partial differential equations resulting from the requirement of harmonic mapping are simple. To this end we first note that the last two terms in Eq. (1) describe a space of constant negative curvature but the line element is not in the canonical form. So we transform to new coordinates $\nu, \omega$ such that

$$
\begin{aligned}
& \cosh \omega=\cosh V \cosh W, \\
& \cos \nu=\left(1+\sinh ^{2} V \operatorname{coth}^{2} W\right)^{-1 / 2},
\end{aligned}
$$

and pass on to Klein's representation of the space of constant curvature by defining

$$
\eta=e^{i \nu} \tanh \frac{1}{2} \omega
$$

On $M$, it is convenient to choose new coordinates by the transformation

$$
\begin{aligned}
& \tau=u\left(1-v^{2}\right)^{1 / 2}+v\left(1-u^{2}\right)^{1 / 2}, \\
& \sigma=u\left(1-v^{2}\right)^{1 / 2}-v\left(1-u^{2}\right)^{1 / 2}
\end{aligned}
$$

which up to a conformal factor brings the line element (10) to a form similar to the standard expression in prolate spheroidal coordinates. ${ }^{11}$ The conformal factor itself is irrelevant because it washes out of the energy functional (8) when $M$ is two dimensional. In the variables (13) and the coordinates (14) the solution is very straightforward. The space-time metric for two colliding impulsive waves is then given by

$$
d s^{2}=2 \frac{1-k \bar{k}}{t r w} d u d v-\frac{t^{2}}{1-k \bar{k}}[(1-k) d x+i(1+k) d y][(1-\bar{k}) d x-i(1+\bar{k}) d y]
$$


where bars denote complex conjugation and we have used the definitions

$$
t^{2}=1-p^{2}-q^{2}, \quad r^{2}=1-p^{2}, \quad w^{2}=1-q^{2},
$$

and

$$
k=e^{i \alpha} p w+e^{i \beta} q r .
$$

We note that in regions II and III Eq. (15) reduces to the desired form [cf. Eqs. (5)] and for $\alpha=\beta=0$ the results of Ref. 6 are obtained. The solution has a curvature singularity on the open interval $\left\{t^{2}=0 ; u>0, v>0\right\}$. We have also found new exact solutions for colliding gravitational waves which in the limit of collinear polarization reduce to the Szekeres family. ${ }^{12}$ These will be published elsewhere.

This work was done mostly while we were visiting the University of Texas at Austin. We are grateful to Professor J. A. Wheeler for advice, encouragement, and his generous hospitality. We are also grateful to Professor S. Chandrasekhar for his kind interest in this work. We thank Dr. R. Güven for many interesting conversations. This work was supported by a NATO Research Grant No. 1002 and a National Science Foundation Grant No. PHY-76-82662.

\footnotetext{
${ }^{1} \mathrm{~J}$. Eells, Jr., and J. H. Sampson, Am. J. Math. $\underline{86}$, 109 (1964).
}

${ }^{2} Y$. Nutku, Ann. Inst. Henri Poincaré A21, 175 (1974).

${ }^{3}$ A. Eris and Y. Nutku, J. Math. Phys. (N.Y.) 16, 1431 (1975); A. Eris, J. Math. Phys. (N.Y.) 18, 824 (1977).

${ }^{4} \mathrm{R}$. Penrose, in General Relativity; Papers in Honor of J. L. Synge, edited by O'Raifeartaigh (Oxford Univ. Press, Oxford, 1972).

${ }^{5} \mathrm{~J}$. Ehlers and W. Kundt, in Gravitation, An Introduction to Current Research, edited by L. Witten (Wiley, New York, 1962).

${ }^{6} \mathrm{~K}$. Khan and R. Penrose, Nature (London) 229, 185 (1971).

${ }^{7} \mathrm{P}$. Szekeres, Nature (London) 228, 1183 (1970).

${ }^{8}$ P. Szekeres, J. Math. Phys. (N. Y.) 13, 286 (1972).

${ }^{9} \mathrm{R}$. Penrose, in Perspectives in Geometry and Relativity, edited by B. Hoffmann (Indiana Univ. Press, Bloomington, 1966).

${ }^{10} \mathrm{C}$. W. Misner, in Magic without Magic: John Archibald Wheeler; A Collection of Essays in Honor of His 6oth Birthday, edited by J. R. Klauder (Freeman, San Francisco, 1972).

${ }^{11} \mathrm{~A}$ geometrical picture of transformations (14) emerges if we consider a square which is to be covered by ellipses with principal axes lying along the diagonals and tangent to the boundary of the square. The interaction region is one quarter of this square where the perpendicular bisector of the sides are the $u, v$ axes and the loci of constant $\tau$ or $\sigma$ are portions of these ellipses. For $\sigma=0$ and $\tau=0$ we obtain two orthogonal straight lines but in the limit of $\sigma \rightarrow 1$ and $\tau \rightarrow 1$ both sets of ellipses degenerate into the circle inscribed by the square and this coincides with the eventual singularity of the solution.

${ }^{12} \mathrm{M}$. Halil, thesis, Middle East Technical University, 1977 (unpublished).

\title{
Solitonlike Properties of Heat Pulses
}

\author{
T. Schneider and E。 Stoll \\ IBM Zurich Research Laboratory, 8803 Rüschlikon, Switzerland
}

and

\author{
Y. Hiwatari \\ Seminar für Theoretische Physik, Eidgenössische Technische Hochschule-Hönggerberg, \\ 8093 Zürich, Switzerland \\ (Received 21 June 1977)
}

\begin{abstract}
Using the molecular-dynamics technique, we establish solitonlike properties of heat pulses in a lattice-dynamic model when the ambient temperature is in the second-sound regime.
\end{abstract}

In this Letter we report on molecular-dynamics results in which we have observed propagation of heat pulses under conditions in which solitonlike properties are established. Recently, considerable effort has been devoted to the study of strong anharmonicity, and a variety of interesting effects are predicted, ${ }^{1-6}$ including optic-mode second sound. ${ }^{7}$

Our present study is performed in a regime where the equilibrium system exhibits second sound, and we use the heat-pulse method. We consider an incompressible model system, where acoustic modes are absent. It belongs to the family of models which have been used with remarkable success to elucidate the critical properties associated with distortive phase transitions. ${ }^{8}$

In this Letter, we first define the model; then we describe our heat-pulse technique and the 